\title{
Valorization of the Phosphate Fertilizers Catalytic Activity in 1-(Benzothiazolylamino) Methyl-2-Naphthol Derivatives Synthesis
}

\author{
Omar Zimou, Badr Malek, Achraf Elhallaoui, Tourya Ghailane, Rachida Ghailane, \\ Said Boukhris, Nouzha Habbadi, Amina Hassikou, Abdelaziz Souizi* \\ Laboratory of Organic, Organometallic and Theoretical Chemistry, Faculty of Sciences, \\ Ibn Tofail University, B.P. 133, Kenitra, Morocco
}

Received: 19th July 2018; Revised: 27th October 2018; Accepted: $4^{\text {th }}$ November 2018; Available online: 30th April 2019; Published regularly: 1st August 2019

\section{Abstract}

The present work focused on developing a new protocol of the 1-(benzothiazolylamino) methyl-2naphthol derivatives synthesis by condensation of three compounds, i.e. aromatic 2-naphthol, 2aminobenzothiazole and aldehyde. Furthermore, this reaction was carried out in the presence of three heterogeneous phosphate catalysts: monoammonium phosphates (MAP), diammonium phosphate (DAP), and trisuperphosphate (TSP). Moreover, this method offered many advantages, such as: very high yields, shorter reaction times, and the catalysts, can be easily recovered and reused without any loss of their catalytic activities. Copyright (C) 2019 BCREC Group. All rights reserved

Keywords: 1-(benzothiazolyamino) methyl-2-naphthol derivatives; Heterogeneous catalysts; Phosphate fertilizers; MAP; DAP; TSP

How to Cite: Zimou, O., Malek, B., Elhallaoui, A., Ghailane, T., Ghailane, R., Boukhris, S., Habbadi, N., Hassikou, A., Souizi, A. (2019). Valorization of the Phosphate Fertilizers Catalytic Activity in 1(Benzothiazolylamino) Methyl-2-Naphthol Derivatives Synthesis. Bulletin of Chemical Reaction Engineering \& Catalysis, 14 (2): 238-246 (doi:10.9767/bcrec.14.2.2976.238-246)

Permalink/DOI: https://doi.org/10.9767/bcrec.14.2.2976.238-246

\section{Introduction}

In the last decades, organic synthesis has been oriented toward the catalysts used to minimize cost, time and energy. One of the most important methods is one-pot multi-component reactions (MCRs) allowing the synthesis of the large variety of compounds that present some activities in medicine domain [1-2].

The compounds containing benzothiazoles at the basic structure are very interesting because they possess biological activities. In particular,

* Corresponding Author.

Email: souizi@yahoo.com (A. Souzi)
2 -aminobenzothiazole is mentioned which is widely used in the fields of medicinal chemistry and biology [3]. They present anti-inflammatory [4], anti-fungal [5] antibacterial [6], anticonvulsant [7], anticancer [8], antitumor [9] activities. They also found their application as indicators of Alzheimer's disease [10], and they are served as reactive and hair dyes [11-12]. Consequently, several processes for the synthesis of 1-(benzothiazolylamino) methyl-2-naphthol using catalysts have been reported in the literature, such as LiCl [13], sodium dodecyl sulphate [14], 3-methyl-1-(4-sulfonic acid) propylimidazo$\mathrm{l} \mathrm{i} \mathrm{m} \quad \mathrm{h} \mathrm{y} \mathrm{d} \mathrm{r} \mathrm{o} \mathrm{g} \mathrm{e} \mathrm{n} \quad \mathrm{s} \mathrm{u} \mathrm{l} \mathrm{f} \mathrm{a} \mathrm{t} \mathrm{e}$ $\left(\left[\left(\mathrm{CH}_{2}\right)_{3} \mathrm{SO}_{3} \mathrm{HMIM}\right]\left[\mathrm{HSO}_{4}\right]\right)[15]$, and heteropoly- 
acid (HPA) [16]. The most of these catalysts present some inconvenient since they are toxic, expensive and non-recyclable. Taking in consideration all these factors, the aim of this study is to propose a new, simple, and effective method for the synthesis of 1-(benzothiazolylamino) methyl-2-naphthol derivatives using phosphate fertilizers monoammonium phosphates (MAP), diammonium phosphate (DAP) and triple superphosphate (TSP) [17] as natural and cheap catalysts, which are well known for their valuable catalytic activities and high recycling and reuse capacity [18-19].

\section{Materials and Method}

All chemicals used were purchased from SIGMA-ALDRICH or FLUKA CHEMICAL. All synthesized products have been identified by comparing their melting points and their spectral data with those of the literature. Melting points were determined on a Kofler bench apparatus and were not corrected. ${ }^{1} \mathrm{H}$ NMR and ${ }^{13} \mathrm{C}$ NMR spectra were recorded on a Bruker FT-NMR spectrometer in DMSO-d6 used as a solvent and TMS as a standard. The progress of

Table 1. 1-((Benzothiazol-2-ylamino) (phenyl) methyl) naphthalen-2-ol synthesisa

\begin{tabular}{cccc}
\hline Entry & Catalyst & Time $(\mathrm{min})$ & Yield $\%^{\mathrm{b}}$ \\
\hline 1 & - & 240 & Traces \\
2 & MAP & 75 & 94 \\
3 & DAP & 35 & 95 \\
4 & TSP & 80 & 95 \\
\hline
\end{tabular}

the reactions was verified and monitored by TLC on silica gel.

2.1 General Procedure for The 1-(benzothiazoly -lamino)phenylmethyl-2-naphthols Synthesis

In a flask of $100 \mathrm{~mL}, 1 \mathrm{mmol}$ of aromatic aldehyde (0.106 g), $1 \mathrm{mmol}$ of 2-naphthol (0.144 $\mathrm{g})$, and $1 \mathrm{mmol}$ of 2 -amino-benzothiazole $(0.150$ g) were added in presence of the catalysts (1 $\mathrm{mg}$ of MAP in $2 \mathrm{~mL}$ of $\mathrm{CHCl}_{3}$ or $1 \mathrm{mg}$ DAP in 2 $\mathrm{mL}$ of cyclohexane or TSP in $2 \mathrm{~mL}$ of THF). The whole was brought to reflux. The progress of the reaction was monitored by TLC. After completion of the reaction, the mixture was washed with acetone and filtered to recover the catalyst which could be reused three times at least and then the filtrate was evaporated and treated with $\mathrm{MeOH}$. The solid obtained was recrystallized from EtOH to give the pure product.

\section{Results and Discussion}

The synthesis of 1-((benzothiazol-2-ylamino)(phenyl) methyl) naphthalen-2-ol was carried out by condensation of benzaldehyde, 2-naphthol, and 2-aminobenzothiazole. This synthesis is considered as a model reaction to optimize the reaction conditions namely solvent nature the amount of catalyst and reaction time. First, the catalytic test was performed using MAP, DAP and TSP catalysts respectively in the presence of chloroform, cyclohexane and tetrahydrofuran; the reaction mixture was refluxed (Table 1).

From the results shown in Table 1, it was noticeable that in the absence of the catalyst, the reaction led to the desired product only in the form of traces. However, using MAP, DAP

Table 2. The solvent effect on the 1-(benzothiazolyamino) methyl-2-naphthol synthesisa

\begin{tabular}{|c|c|c|c|c|c|c|c|}
\hline \multirow[b]{2}{*}{ Entry } & \multirow[b]{2}{*}{ Solvent } & \multicolumn{2}{|c|}{ MAP } & \multicolumn{2}{|c|}{ DAP } & \multicolumn{2}{|c|}{ TSP } \\
\hline & & $\begin{array}{l}\text { Time } \\
\text { (min) }\end{array}$ & $\begin{array}{c}\text { Yield } \\
\%^{\mathrm{b}}\end{array}$ & $\begin{array}{l}\text { Time } \\
\text { (min) }\end{array}$ & $\begin{array}{c}\text { Yield } \\
\%^{\mathrm{b}}\end{array}$ & $\begin{array}{l}\text { Time } \\
\text { (min) }\end{array}$ & $\begin{array}{c}\text { Yield } \\
\%^{\mathrm{b}}\end{array}$ \\
\hline 1 & $\mathrm{EtOH}$ & 85 & 65 & 55 & 66 & 100 & 67 \\
\hline 2 & $\mathrm{CH}_{3} \mathrm{CN}$ & 65 & 78 & 65 & 68 & 80 & 73 \\
\hline 3 & $\mathrm{MeOH}$ & 95 & 72 & 45 & 63 & 90 & 66 \\
\hline 4 & $\mathrm{THF}$ & 80 & 86 & 55 & 82 & 90 & 98 \\
\hline 5 & $\mathrm{CHCl}_{3}$ & 85 & 98 & 45 & 89 & 95 & 93 \\
\hline 6 & Cyclohexane & 78 & 89 & 50 & 98 & 85 & 87 \\
\hline 7 & Solvent-free & 90 & 60 & 65 & 60 & 100 & 62 \\
\hline
\end{tabular}

a Reaction of 2-naphthol $(1 \mathrm{mmol})$ 2-aminobenzothiazole $(1 \mathrm{mmol})$ and benzaldehyde $(1 \mathrm{mmol})$ catalyzed by MAP, DAP and TSP under reflux conditions.

${ }^{b}$ Isolated yield. 
and TSP as catalysts in the reaction medium, the excellent yields of $98 \%$ were obtained in short reaction times ranging between 50 and 90 min. This result indicates that the catalysts used here possess an interesting catalytic activity for the synthesis taken as a model reaction.

It is well known that the yields and the reaction time also depend on other parameters such as the nature of the solvent and the amount of catalyst. For this reason, the reaction conditions for each catalyst it were optimized. First, the solvent effect, on the yield and the reaction time, has been studied. The model reaction was performed without solvent and in the presence of different solvents, namely the apolar aprotic protic polar $(\mathrm{MeOH}, \mathrm{EtOH})$.

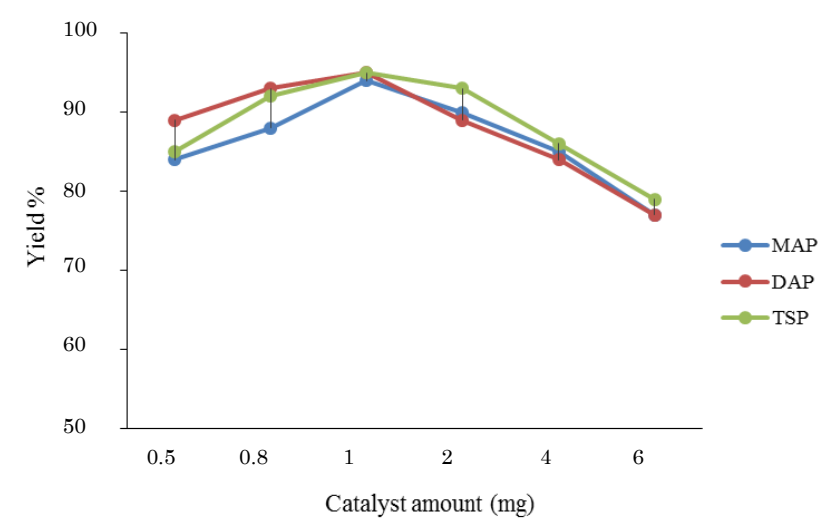

Figure 1. Variation of the catalyst amount for the 1-(benzothiazolyamino) methyl-2-naphthol synthesis*.

*Reaction conditions: 2-naphthol (1 mmol) 2-aminobenzothiazole $(1 \mathrm{mmol})$, benzaldehyde $(1 \mathrm{mmol})$ with an amount of catalyst varying from 0.5 to $6 \mathrm{mg}$ and in the presence of $\mathrm{CHCl}_{3}, \mathrm{Cyclohexane}$ and THF as solvents for MAP, DAP, and
(Cyclohexane, $\left.\mathrm{CHCl}_{3}\right)$, polar aprotic $(\mathrm{MeCN}$, THF). Table 2 resumed the obtained yields and the reaction time.

Table 2 shows that the reactions carried out in the absence of the solvent provided the expected product with average yields of about $60 \%$. In contrast, the presence of the solvents improves the yields; this can be explained by the important role played by the solvent to facilitate the interaction between the reagents and the catalyst. According to the yields obtained it is found that the reactions were more easily done in aprotic solvents, $\mathrm{CHCl}_{3}(98 \%)$, Cyclohexane (98\%) and THF (98\%) respectively for MAP, DAP and TSP. Indeed, the aprotic solvents favorite a good charge separation in the reagents and an easy reaction process.

Moreover, the nature of the used solvent and the catalyst amount should be a determinant parameter in the reaction medium. Thus the influence of the amount of catalysts was studied by varying the mass of MAP, DAP, and TSP from $0.5 \mathrm{mg}$ to $6 \mathrm{mg}$. Figure 1 presents the obtained results.

It can be seen, from the Figure 1, that when the catalyst amount increases the yield of the reaction decreases, this is probably due to the dispersion of the reagents on the surface of the catalyst which prevent an easy interaction between the different compounds involved in the reaction. One can deduce that $1 \mathrm{mg}$ of each catalyst is sufficient to give the best yield of the desired product. In term of the gain on the reaction time, the time was varied it by a step of 5 min six times in case of each catalyst. The obtained results are summarized in Table 3.

Table 3 shows that the yield of the product increases gradually with the increasing time. Thus the sufficient times leading to the best

Table 3. Time effect on the yield of the 1- (benzothiazolyamino) methyl-2-naphthol synthesisa

\begin{tabular}{|c|c|c|c|c|c|c|}
\hline \multirow{2}{*}{ Entry } & \multicolumn{2}{|c|}{ MAP } & \multicolumn{2}{|c|}{ DAP } & \multicolumn{2}{|c|}{ TSP } \\
\hline & Time (min) & Yield $(\%)^{b}$ & Time (min) & Yield $(\%)^{b}$ & Time (min) & Yield $(\%)^{b}$ \\
\hline 1 & 65 & 88 & 25 & 88 & 70 & 84 \\
\hline 2 & 70 & 90 & 30 & 90 & 75 & 90 \\
\hline 3 & 75 & 94 & 35 & 95 & 80 & 95 \\
\hline 4 & 80 & 96 & 40 & 97 & 85 & 97 \\
\hline 5 & 85 & 98 & 45 & 97 & 90 & 98 \\
\hline 6 & 90 & 98 & 50 & 98 & 95 & 98 \\
\hline 7 & 95 & 98 & 55 & 98 & 100 & 98 \\
\hline
\end{tabular}

a Reaction of 2-naphthol $(1 \mathrm{mmol})$ 2-aminobenzothiazole $(1 \mathrm{mmol})$ and benzaldehyde $(1 \mathrm{mmol})$ catalyzed by MAP, DAP, and TSP under reflux conditions.

${ }^{b}$ Isolated yield. 
yields are $85 \mathrm{~min}, 50 \mathrm{~min}$, and $90 \mathrm{~min}$ for the MAP (98\%), DAP (98\%), and TSP (98\%), respectively. One can deduce that in the presence of catalysts there is a considerable increase in the yields.

Taking into account the optimized conditions for the pilot reaction, the procedure was applied to prepare some 1-(benzothiazoly-



Scheme 1. 1-((Benzothiazol-2-ylamino)(phenyl) methyl) naphthalen-2-ol synthesis amino) methyl-2-naphthol derivatives, by the condensation of 2-naphthol, 2-aminobenzothiazole using a variety of aromatic aldehyde differently substituted by electro-donor and electro-attractor groups (Scheme 2). The results are collected in Table 4.

From Table 4 it is observed that the yields of the products obtained remain better whatever the substituent of the aromatic cycle of the aldehyde (63-98\%). For the calculated TON and TOF, the best values are obtained for the model reaction in the presence of MAP and DAP, which are, respectively, 108.1/86.4 $\mathrm{h}^{-1}$ and 125.4/216.2 $\mathrm{h}^{-1}$. For TSP, the best values of TON and TOF are observed for the condensation of 4-chlorobenzaldehyde, 2-naphthol and 2 -aminobenzothiazole $\left(229.5 / 153.0 \mathrm{~h}^{-1}\right)$.

Table 4. Generalization of the Synthesis of 1- (benzothiazolyamino) methyl-2-naphthol derivatives ${ }^{\text {a }}$

\begin{tabular}{|c|c|c|c|c|c|c|c|c|}
\hline \multirow{2}{*}{ Entry } & \multirow{2}{*}{$\mathrm{R}-\mathrm{CHO}$} & \multirow{2}{*}{ Catalyst } & \multirow{2}{*}{$\begin{array}{l}\text { Time } \\
(\mathrm{min})\end{array}$} & \multirow{2}{*}{$\begin{array}{l}\text { Yield } \\
\left(\%^{b}\right)\end{array}$} & \multirow{2}{*}{ TON } & \multirow{2}{*}{$\begin{array}{c}\text { TOF } \\
\left(\mathrm{h}^{-1}\right)\end{array}$} & \multicolumn{2}{|c|}{ Melting point $\left({ }^{\circ} \mathrm{C}\right)$} \\
\hline & & & & & & & Found & $\begin{array}{c}\text { Reported } \\
\text { [References] }\end{array}$ \\
\hline \multirow{3}{*}{$4 \mathrm{a}$} & & MAP & 75 & 94 & 108.1 & 86.4 & \multirow{3}{*}{$201-203$} & \multirow{3}{*}{ 202-204 [20] } \\
\hline & & DAP & 35 & 95 & 125.4 & 216.2 & & \\
\hline & & TSP & 80 & 95 & 222.5 & 167.3 & & \\
\hline \multirow{3}{*}{$4 \mathrm{~b}$} & & MAP & 150 & 87 & 100.1 & 40.1 & \multirow{3}{*}{$208-210$} & \multirow{3}{*}{$209-210[21]$} \\
\hline & & DAP & 45 & 90 & 118.9 & 158.5 & & \\
\hline & & TSP & 90 & 98 & 229.5 & 153.0 & & \\
\hline \multirow{3}{*}{$4 \mathrm{c}$} & & MAP & 180 & 86 & 98.9 & 32.9 & \multirow{3}{*}{$210-211$} & \multirow{3}{*}{$200-202[21]$} \\
\hline & & DAP & 80 & 82 & 97.7 & 73.4 & & \\
\hline & & TSP & 120 & 80 & 187.4 & 93.6 & & \\
\hline \multirow{3}{*}{$4 \mathrm{~d}$} & & MAP & 150 & 74 & 86.3 & 34.5 & \multirow{3}{*}{$183-185$} & \multirow{3}{*}{$182-183[13]$} \\
\hline & & DAP & 85 & 72 & 95.1 & 67.4 & & \\
\hline & & TSP & 100 & 63 & 147.5 & 88.8 & & \\
\hline \multirow{3}{*}{$4 \mathrm{e}$} & & MAP & 165 & 76 & 87.4 & 31.7 & \multirow{3}{*}{$173-175$} & \multirow{3}{*}{$172-173[20]$} \\
\hline & & DAP & 90 & 74 & 99.1 & 66.1 & & \\
\hline & & TSP & 120 & 67 & 156.9 & 78.4 & & \\
\hline \multirow{3}{*}{$4 \mathrm{f}$} & & MAP & 165 & 78 & 89.7 & 32.6 & \multirow{3}{*}{$175-177$} & \multirow{3}{*}{$176-177[16]$} \\
\hline & & DAP & 60 & 75 & 99.0 & 99.0 & & \\
\hline & & TSP & 155 & 77 & 180.3 & 69.8 & & \\
\hline
\end{tabular}

a Reaction of 2-naphthol $(1 \mathrm{mmol})$ 2-aminobenzothiazole $(1 \mathrm{mmol})$ and benzaldehyde $(1 \mathrm{mmol})$ catalyzed by MAP, DAP, and TSP under reflux conditions.

${ }^{b}$ Isolated yield. 
The spectral data for all products and the spectra of ${ }^{1} \mathrm{H}$ NMR and ${ }^{13} \mathrm{C}$ NMR for the product obtained in the model reaction are given below:

1-((Benzo[d]thiazol-2-ylamino)(phenyl) methyl)naphthalen-2-ol (Table 4. 4a, Figures 3 and 4). $\mathrm{mp}: 202-204^{\circ} \mathrm{C} .{ }^{1} \mathrm{H}$ NMR $(300 \mathrm{MHz}$, DMSO-d 6 ): $\delta$ (ppm): 6, 97-7.88 (m. 16H. ArH and $\mathrm{CH}), 8.79$ (s. $1 \mathrm{H} . \mathrm{NH}), 10.16$ (s. $1 \mathrm{H} . \mathrm{OH})$; ${ }^{13} \mathrm{C}$ NMR (300 MHz, DMSO-d 6 ): $\delta$ (ppm): 53.58, $118.57,118.88,119.18,121.35,121.46,122.88$,
$124.13,125.90,126.53,126.65,128.57,129.02$, $129.12,130.03,131.26,142.98,152.59,153.67$, 166.78 .

1-((Benzo[d]thiazol-2-ylamino)(4-chloro phenyl) methyl)naphthalen-2-ol (Table 4. 4b). mp: 209-210 ${ }^{\circ}$ C. ${ }^{1} \mathrm{H}$ NMR (300 MHz, DMSO-d 6 ): $\delta$ (ppm): 6, 98-7.80 (m. 15H. ArH and CH), 8. 82 (s. $1 \mathrm{H} . \mathrm{NH}), 10.21$ (s. $1 \mathrm{H} . \mathrm{OH}) ;{ }^{13} \mathrm{C}$ NMR $(300$ MHz, DMSO- $\left.\mathrm{d}_{6}\right): \delta$ (ppm): $53.11,118.72$, $118.86,121.39,121.57,122.97,124.10,125.93$, $126.87,128.42,128.53,159.10,130.27,131.19$,

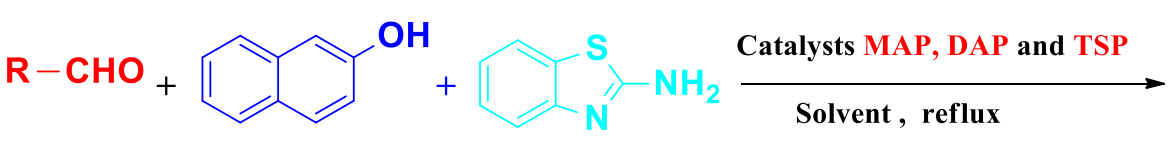
1a-f 2 3<smiles>[R]C(Nc1nc2ccccc2s1)c1c(O)ccc2ccccc12</smiles>

$4 a-f$

Scheme 2. Synthesis of 1-(benzothiazolyamino) methyl-2-naphthol derivatives
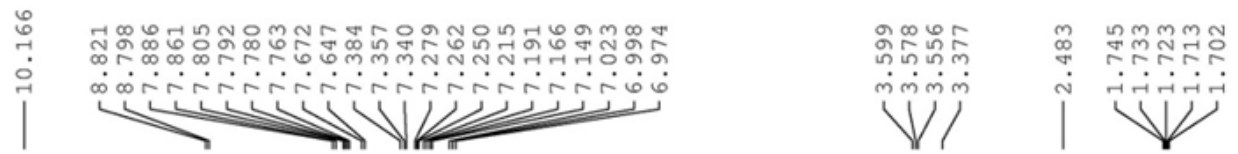<smiles>Oc1ccc2ccccc2c1C(Nc1nc2ccccc2s1)c1ccccc1</smiles>

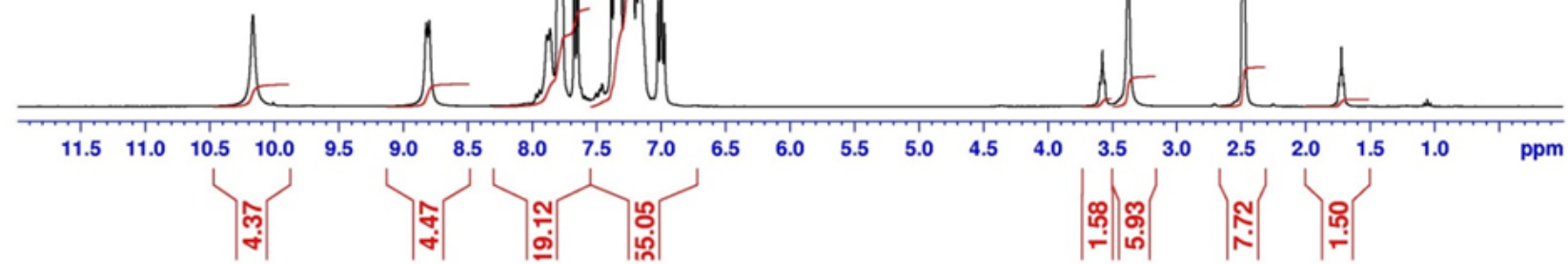

Figure 2. ${ }^{1} \mathrm{H}$ NMR of 1-((Benzo[d]thiazol-2-ylamino)(phenyl)methyl)naphthalen-2-ol (4a) in DMSO-d 6 
131.33, 132.52, 142.19, 152.53, 153.71, 166.68.

1-((Benzo[d]thiazol-2-ylamino)(4-bromo phenyl) methyl)naphthalen-2-ol (Table 4. 4c). mp: 209$210^{\circ} \mathrm{C} .{ }^{1} \mathrm{H}$ NMR (300 MHz, DMSO-d 6$): \delta(\mathrm{ppm})$ : 6, 97-7.81 (m. 15H. ArH and CH), 8. 79 (s. 1H. $\mathrm{NH}), 10.18$ (s. $1 \mathrm{H} . \mathrm{OH})$; ${ }^{13} \mathrm{C} \mathrm{NMR}(300 \mathrm{MHz}$, DMSO- $\left.\mathrm{d}_{6}\right): \delta(\mathrm{ppm}): \quad 53.10,118.64,118.68$, 118.82 , 119.61, 121.39, 121.56, 122.97, 124.10, $125.91,121.87,128.79,129.09$, 130.28, 131.32, 131.43, 132.48, 142.65, 152.51, 153.67, 166.64 .

1-((Benzo[d]thiazol-2-ylamino)(4-methyl phenyl)methyl)naphthalen-2-ol (Table 4. 4d). mp: $183-185^{\circ} \mathrm{C}$. ${ }^{1} \mathrm{H}$ NMR (300 MHz, DMSO-d 6$): \delta$ (ppm): $2.21\left(\mathrm{~s}, 3 \mathrm{H}, \mathrm{CH}_{3}\right), 6,96-7.87$ (m. $15 \mathrm{H}$. ArH and CH), 8.76 (s. 1H. NH), 10. 13 (s. $1 \mathrm{H}$. $\mathrm{OH}) ;{ }^{13} \mathrm{C}$ NMR (300 MHz, DMSO-d 6$): \delta$ (ppm): 21.03, 53.43, 118.53, 118.88, 119.27, 121.34, $121.42,122.85,124.42,125.90,126.48,126.62$, 128.99 , 129.15, 129.94, 131.22, 132.60, 135.61, 139.89, 152.61, 153.61, 166.77 .

1-((Benzo[d]thiazol-2-ylamino)(4-methoxy phenyl) methyl)naphthalene-2-ol (Table 4. 4e). mp:
173-175 ${ }^{\circ}$. ${ }^{1} \mathrm{H}$ NMR (300 MHz, DMSO-d 6$): \delta$ (ppm): $3.66\left(\mathrm{~s}, 3 \mathrm{H}, \mathrm{OCH}_{3}\right), 6,80-7.88$ (m. $15 \mathrm{H}$. $\mathrm{ArH}$ and $\mathrm{CH}$ ), 8.74 (s. $1 \mathrm{H}$. NH), 10. 11 (s. $1 \mathrm{H}$. $\mathrm{OH}) ;{ }^{13} \mathrm{C}$ NMR (300 MHz, DMSO-d 6$): \delta$ (ppm): 53.25 , 55.45, 114.00, 118.51, 118.88, 119.22, $121.33,121.39,122.84,124.35,125.89,126.60$, $127.71,128.99,129.12,129.90,131.18,132.59$, $134.63,152.61,153.57,158.19,166.730$.

1 - (Benzo[d] thiazol- 2 -ylamino)(furan - 2 yl)methyl)naphthalene-2-ol ( Table 4. 4f). mp: $176-177^{\circ} \mathrm{C}$. ${ }^{1} \mathrm{H}$ NMR (300 MHz, DMSO-d 6 ): $\delta$ (ppm): 6,19-8.89 (m. 14H. ArH and CH), 8. 91 (s. $1 \mathrm{H} . \mathrm{NH}), 10.23$ (s. $1 \mathrm{H} . \mathrm{OH}) ;{ }^{13} \mathrm{C} \mathrm{NMR}(300$ $\mathrm{MHz}$, DMSO-d6): $\delta$ (ppm): 49.17, 106.87, $110.96,116.65,118.63,118.90,121.39,121.54$, $122.92,123.85,125.96,126.73,128.98,130.26$, $131.14,132.86,142.34,152.44,153.92,155.00$, 166.32 .

To obtain these products we proposed the mechanism presented in Scheme 3. In the reaction medium, the catalysts MAP, DAP, and TSP having an acid character, release a proton which activates the carbonyl group of the aldehyde, the latter reacts with 2-naphthol to give

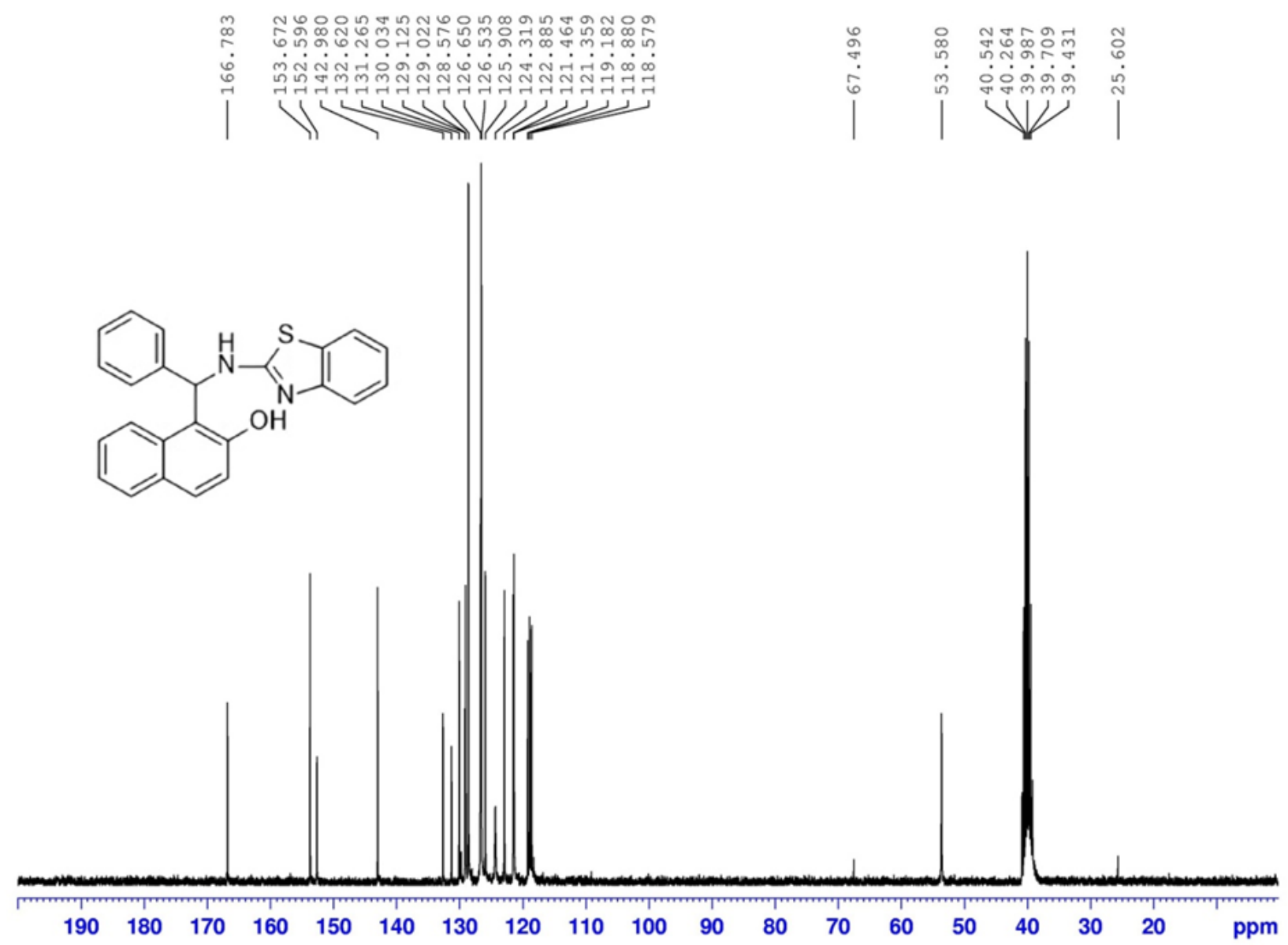

Figure 3. ${ }^{13} \mathrm{C}$ NMR of 1-((Benzo[d]thiazol-2-ylamino)(phenyl)methyl)naphthalen-2-ol (4a) in DMSO-d 6 
the o-QM, then the catalysts activate the carbonyl of ortho-Quinone methides (o-QMs) which will subsequently react in situ with 2 aminobenzothiazole to form the desired products $4 \mathrm{a}-\mathrm{f}$.

In order to test the long-term durability of MAP, DAP and TSP. We studied the reuse of

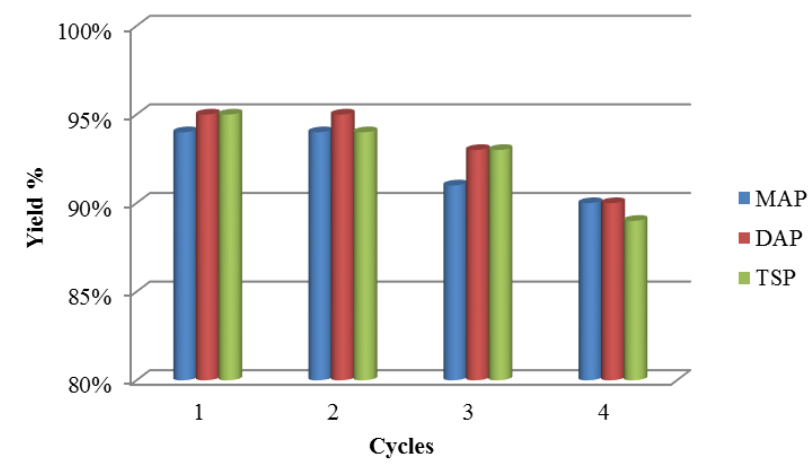

Figure 4. Recycling of MAP, DAP and TSP in case of the 1-(benzothiazolyamino) methyl-2naphthol synthesis these four catalysts for the synthesis of 1((Benzothiazol-2-ylamino) (phenyl) methyl) naphthalen-2-ol, at the end of each reaction the catalysts were recovered by simple filtration, washed with $\mathrm{EtOH}$, then dried in the oven at $200{ }^{\circ} \mathrm{C}$ for one hour, they are then reused for three times. Figure 4 shows the results obtained.

From Figure 4, it can be seen that after four cycles, a slight loss of catalytic activity is observed. Therefore, the phosphate fertilizers used herein can be considered as interesting and efficient catalysts in the synthesis of 1-(benzothiazolyamino) methyl-2-naphthol derivatives.

\section{Conclusion}

In this work, a simple and efficient method has been developed by the application of phosphate fertilizers MAP, DAP, and TSP as heterogeneous catalysts for the synthesis of 1-(benzothiazolylamino) methyl-2-naphthol. The optimization of the operating conditions

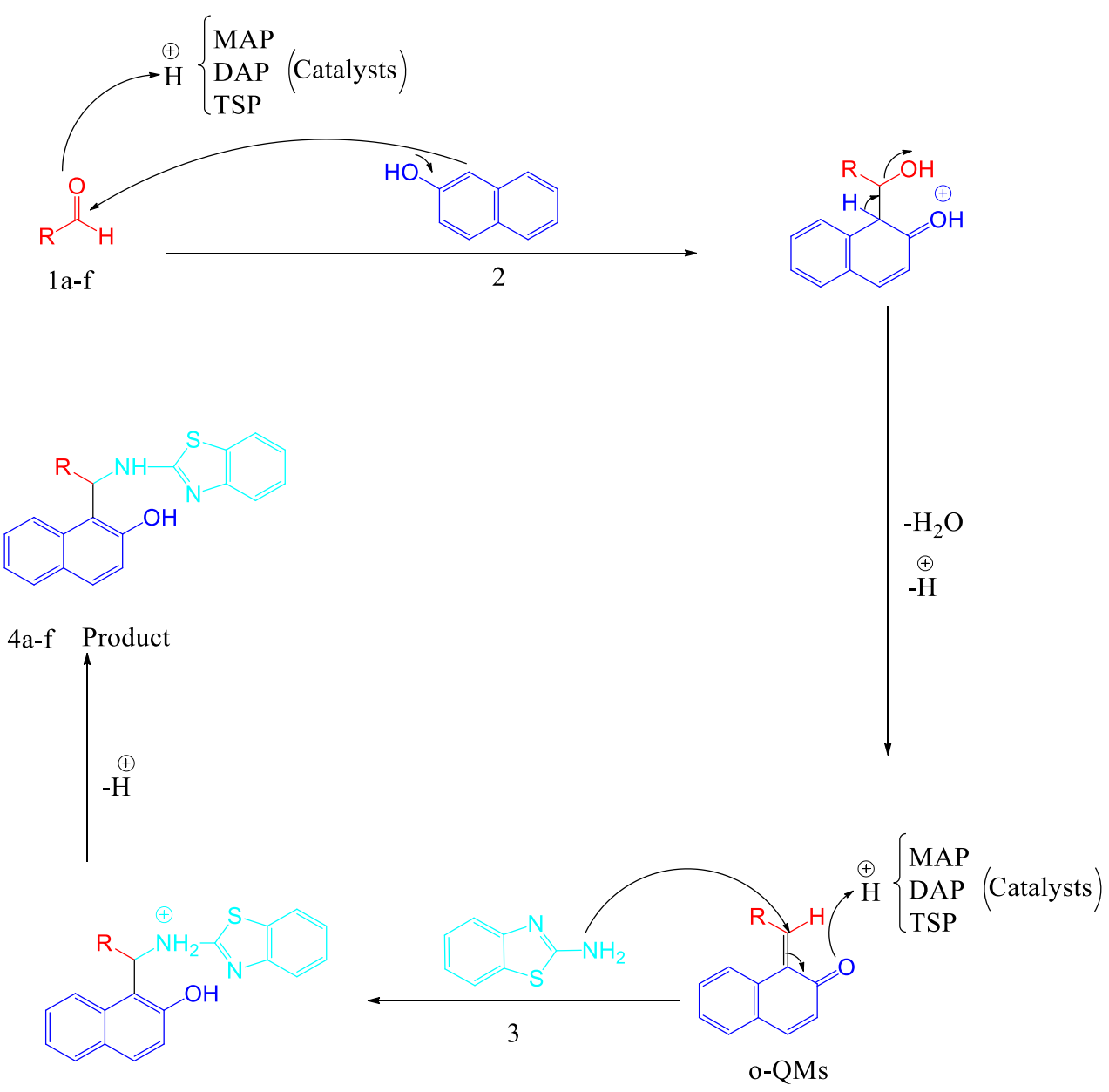

Scheme 3. Suggested mechanism for the synthesis of 1-(benzothiazolyamino) methyl-2-naphthol derivatives by MAP, DAP, and TSP catalysts 
such as solvent, a catalyst mass and reaction time to facilitate the obtaining of the desired products with higher yields in short reaction times. Also, these catalysts show a good reutilizing capacity such that they can be recovered and reused several times after the first use without loss of their catalytic activities, which is shown the catalytic efficiency of them. The novelty of this work appears in the fact that the used catalysts are not toxic, available, and not expensive. Moreover, the present research can contribute to the valorization of the natural phosphate because Morocco is considered a leading producer of phosphate products.

\section{Acknowledgement}

The authors thank the Center for Analysis, Expertise, Technology Transfer and Incubation (CUAE2TI) of Ibn Tofail University.

\section{References}

[1] Xu, L. W., Xia, C. G., Li, L. (2004). Transition Metal Salt-Catalyzed DirectThree-Component Mannich Reactions of Aldehydes, Ketones, and Carbamates: Efficient Synthesis of NProtected B-Aryl-B-Amino Ketone Compounds. J. Org. Chem., 69: 8482-8484.

[2] Dömling, A. (2006). Recent Developments in Isocyanide Based Multicomponent Reactions in Applied Chemistry. Chem. Rev., 106: 17-89.

[3] Katritzky, A.R., Tymoshenko, D.O., Monteux, D., Vvedensky, V., Nikonov, G., Cooper, C.B., Deshpande, M. (2000). A New Three-Carbon Synthon for Efficient Synthesis of Benzannelated and 1-(2-Arylethenyl) Heterocycles. $J$. Org. Chem., 65: 8059-8062.

[4] El-Shorbagi, A. N., Sakai, S., El-Gendy, M.A., Omar, N., Farag, H. H. (1989). Imidazo(2,1b)benzothiazoles. II. Synthesis and antiinflammatory activity of some imidazo(2,1b)benzothiazoles. Chem. Pharm. Bull., 37: 2971-2975.

[5] Yang, X., Liang, J. (2011). 1,3-Dibromo-5,5dimethylhydantoin catalyzed one-pot synthesis of 2-arylbenzothiazoles. Collect. Czechoslov Chem. Commun., 76: 1909-1915.

[6] Palkar, M., Noolvi, M., Sankangoud, R., Maddi, V., Gadad, A., Nargund, L.V.G. (2010). Synthesis and Antibacterial Activity of a Novel Series of 2,3-Diaryl-substitutedimidazo(2,1-b) benzothiazole Derivatives. Arch. Pharm., 343: 353-359.

[7] Chopade, R.S., Bahekar, R.H., Khedekar, P.B., Bhusari, K.P., Ram Rao, A.R. (2002). Synthesis and Anticonvulsant Activity of 3-(6Substituted-benzothiazol-2-yl)-6-phenyl-[1,3]xazinane-2-thiones. Arch. Pharm., 335: 381-
388.

[8] Yang, Z., Wang, A., Chen, X., Gui, Q., Liu, J., Tan, Z. (2013). Synthesis of 2-Aryl Benzothiazoles via $\mathrm{K}_{2} \mathrm{~S}_{2} \mathrm{O}_{8}$-Mediated Oxidative Condensation of Benzothiazoles with Benzylamines. Synlett., 24: 1549-1554.

[9] Sadhasivam, G., Kulanthai, K., Natarajan, A. (2015). Synthesis and Anti-cancer Studies of 2, 6-disubstituted Benzothiazole Derivatives. Orient. J. Chem., 31: 819-826.

[10] Sharma, A.K., Kim, J., Prior, J.T., Hawco, N.J., Rath, N. P., Kim, J. (2014). Small Bifunctional Chelators That Do Not Disaggregate Amyloid B Fibrils Exhibit Reduced Cellular Toxicity. Inorg. Chem., 53: 11367-11376.

[11] Lashkari, M., Maghsoodlou, M.T., Karima, M., Adrom, B., Fatahpour, M. (2016). Convenient Approach for the One-Pot, ThreeComponent Synthesis of 1 (Benzothiazolylamino) Methyl-2-Naphthol Using Citric Acid as a Green Catalyst. Acta Chem. Iasi., 24: 212-221.

[12] Adrom, B., Maghsoodlou, M.T., Hazeri, N., Lashkari, M. (2015). Solvent-free synthesis of 1-(benzothiazolylamino)methyl-2-naphthols with maltose as green catalyst. Res. Chem. Intermed., 41: 7553-7560.

[13] Shaabani, A., Rahmati, A., Farhangi, E. (2007). Water promoted one-pot synthesis of 2 -aminobenzothiazolomethyl naphthols and 5 - ( $2^{\prime}$ - a minobenzothiazolomethyl) - 6 hydroxyquinolines. Tetrahedron Lett., 48: 7291-7294.

[14] Kumar, A., Rao, M.S., Rao, V.K. (2010). Sodium Dodecyl Sulfate-assisted Synthesis of 1(Benzothiazolylamino)methyl-2-naphthols in Water. Aust. J. Chem., 63: 1538-1540.

[15] Shaterian, H.R., Hosseinian, A.A. (2015). Brønsted acidic ionic liquid, $\left[\left(\mathrm{CH}_{2}\right)_{3} \mathrm{SO}_{3} \mathrm{HMIM}\right]\left[\mathrm{HSO}_{4}\right]$, as an efficient catalyst for synthesis of 1 (benzothiazolylamino) methyl-2-naphthols. Res. Chem. Intermed., 41: 793-801.

[16] Maghsoodlou, M.T., Karima, M., Lashkari, M., Adrom, B., Aboonajmi, J. (2017). A green protocol for one-pot three-component synthesis of 1-(benzothiazolylamino) methyl-2naphthol catalyzed by oxalic acid. J. Iran Chem., 14: 329-335.

[17] Bahammou, I., Esaady, A., Boukhris, S., Ghailane, R., Habbadi, N., Hassikou, A., Souizi, A. (2016). Direct use of mineral fertilizers MAP, DAP, and TSP as heterogeneous catalysts in organic reactions. Mediterr. J. Chem., 5: 615-623.

[18] Sibous, S., Ghailane, T., Houda, S., Ghailane, R., Boukhris, S., Souizi, A. (2017). Green and efficient method for the synthesis of 1,5- 
benzodiazipines using phosphate fertilizers as catalysts under free solvent. Mediterr. J. Chem., 6: 53-59.

[19] Sibous, S., Boukhris, S., Ghailane, R., Habbadi, N., Hassikou, A., Souizi, A. (2017). Easy synthesis of 3,4-dihydropyrimidin -2-(1H)ones using phosphate fertilizers MAP, DAP and TSP as efficient catalysts. J. Turk Chem. Soc. Sect. Chem., 4: 477-488.

[20] Venkatesan, K., Pujari, S.S., Lahoti, R.J., Srinivasan, K.V. (2008). An efficient synthesis of 1,8-dioxo-octahydro-xanthene derivatives promoted by a room temperature ionic liquid at ambient conditions under ultrasound irradiation. Ultrason. Sonochem., 15: 548-553.
[21] Hosseinian, A., Shaterian, H.R. (2012). Na$\mathrm{HSO}_{4} \cdot \mathrm{H}_{2} \mathrm{O}$ Catalyzed Multicomponent Synthesis of 1-(Benzothiazolylamino) Methyl-2Naphthols Under Solvent-Free Conditions. Phosphorus Sulfur Silicon Relat. Elem., 187: 1056-1063. 